\title{
Ueber cyklisch-projectivische Punktgruppen in der Ebene und im Raume.
}

Von J. Lürota in Karlsrube.

In einer Arbeit, die in XI. Bande dieser Annalen ersehienen ist, habe ich auf geometrischen Wege einge Eigenschaften der cyllischprojectivischen Punktgruppen auf einer geraden Linie abgeleitet. Die gegenwärtige Abhandlung behandelt die allgemeinere frage yach den cyklisch projectivischen Punltyruppen in der Ebene und im Raume und löst die Autgabe: in der Elene (oder im Faume) n relle Punlte $a_{1} a_{2} a_{3} \cdots a_{n}$ so za bestimmen, dass es projectivisehe Transformationen der Ebene (resp. des Raumes) giebt, welche $a_{3}$ in $a_{2}$, $a_{2}$ in $a_{3}, \cdots a_{n}$ in $a$, überfuliren. Ich werde eine projectivische Transformation der Ebene oder des Raumes (und nur von projectivischen Trapsformationen ist im Folgenden die Rede), welche die Punkte abc... in die Punkte $a^{\prime} b^{\prime} c^{\prime}$... uberfübrt, kiunftig als die Transformation $a b c \ldots ; a^{\prime} l^{\prime} c^{\prime} \ldots$ bezeichnen. Statt des Zeichens $a_{1} a_{2} \ldots ; a_{y+1} a_{p+2} \cdots$ werde ich aber auch die einfacheren $\mid a_{i}, a_{i+p}$ oder $\left.i, i+1\right)$ beniüzen. Hier, wie in Folgenden stets, ist unter einem Index, der $>n$ ist, sein Rest nach dem Modul $n$ zu verstehen. Ich benutze bel den Ableitungen auch imaginäre Elemente, die ich aber stets als solche susdrilcklich bezeichne.

1) Haben die 12 Punkte $a_{1} a_{2} \cdots a_{n}$ eine solche lage, dass eine Transformation $i, i+1=T$ existirt und man wendet dieselbe zweimal an, so entsteht die Transformation $i, i+2=T^{2}$ eine weitere Anwendung liefert die Umformung $i, i+3=T^{3}, u$, s. $w$. , so dass allgemein die Punkte eine Transformation $T=i, i+p$ zulasien. Die Transformation $T^{n}=\mid i, i+n=i$, lässt jeden der Punkte $a_{1}, a_{2} \cdots a_{n}$ noverändert and folglich aberhaupt joden Punkt in der Ebene oder im Raume, wenn man von besonderea, nachher aunzuschliessenden, Lagen der P'unkte absieht. Gesetzt aber es gälue eine Potenz von $T$ mit einem Exponenten $q<n$, welehe $i$, $i$ wïre; daun minsten die Punkte $a_{y+:} a_{1+2} \cdots a_{4}$, welche durch $T^{4}$ ans $a_{3} a_{7} \cdots a_{*}$ 
hervorgehen, der Reihe nach mit $a_{1} a_{2} \cdots a_{n}$ identiseh sein, und es könnten also die Punkte $a_{1} \cdots a_{n}$ nicht alle unter sich verschieden sein. Vielmehr wärden dann nur die Punkte $a_{1} a_{2} \cdots a_{q}$ in der That verschieden sein, und die übrigen mit diesen zusarnmenfallen, so dass $a_{q+1}=a_{1,2} a_{q+2}=a_{2}, \cdots a_{k}=a_{q}$ wäre. Dies verlangt, dass $q$ ein Theiler von $n$ und $n=p q$ ist. Seien umgekehrt vou der Gruppe nur die Puxkte $a_{1} a_{2} \cdots a_{q}$ von einander verscbieden. Durch $T$ gehen aus ihnen die $a_{2} a_{3} \cdots a_{q+1}$ hervor. Wäre nun $a_{q+1}$ nicht mit $a_{1}$, soudern mit einem andern der $q$ Punlte z. B. $a_{i+1}$ identisch, so minssten beide vermöge $T$ aus demselben Punkte liervorgegangen sein $d$. h. es müssten, gegen die Annahme, $a_{q}$ und $a_{i}$ identisch sein. Es muss also $a_{q+1}=a_{i}$ sein und daraus folgt, dass $a_{q+2}=a_{22} \cdots$ allgemein $a_{r q+s}=a_{s}$ sein muss, und dass die Punkte, deren Indices Vielfache von $q$ sind, die einzigen sind, welche durch $T$ in $a_{1}$ übergeführt werden. Da dies von $a_{n}$ angenommen ist, muss $n$ durch $q$ theilbar sein; es hat dem. nach $T$ die Eigensehaft, dass $T^{q}=|\dot{i}, i|$ ist. Dann ist aber $T=\left|a_{1} a_{2} \cdots a_{9} ; a_{2} a_{3} \cdots a_{1}\right|$ und die Punkte $a_{1} a_{2} \cdots a_{i}$ bilden eine cyklisch-projectivische Gruppe. Diese letzteren Betrachtungen gelten ebenso, wenn $a_{1} a_{2} \cdots a_{n}$ Punlte einer Punktreihe, oder Strahlen eines Strahlenbüschels oder Ebenen eines Ebenenbüschels rorstellen, und in der That werden wir auch bei solchen Gebilden davon Gebrauch zu machen haben, während wir für die Hauptaufgabe das Vorkommen dieser Ausnahme dadurch ausschliessen, dass wir annehmen, die zu suchenden Punkte seien alle versehieden von einander.

\section{Panktgrappen in der Ebene.}

2) Da eine projectivische Transformation einer Ebene in sich selbst bestimmt ist, wenn 4 Paare sich entsprechender Punkte gegeben sind, so wässen erst 5 Punkte eine gewisse Bedingung erfüllen, wenn sie eine cykl.-proj. Gruppe bilden sollen und wir schliessen desshalb die Falle $n<4$ hier aus. Wenn nun für die Punkte $a_{1} a_{2} \cdots a_{n}(n \geq 0)$ einer Ebene eine Transformation $T=|i, i+1|$ existirt, so giebt es, wie bei jeder projectivischen Transformation einer Ebene in sich, jedenfalls einen Punkt $A$, und eine Gerade $g$, die in sich selbst übergeführt werden oder Doppelelemente der Transformation sind*). Wenn wir hier und in Folgenden den sehon früher erledigten Fall ausschliessen, dass die ganze Gruppe auf einer Geraden liegt, so kaun $g$ nicht durch $A$ gehen. Dern da vermöge $T$ der Büschel $A\left(a_{1} a_{2} \cdots a_{n}\right) \Uparrow A\left(a_{2} a_{3} \cdots a_{1}\right)$ ist, also die Strahlen $A\left(a_{1} \cdots a_{n}\right)$ eine cykhsch-projectivische Gruppe bilden, so kann kein Strahl von $A$ durch $T$ in sich übergeführt wer-

5) Vergl. v. Standt, Geometrie der Lage. Seite 199, No. 294. 
den, weil sonst die zur Gruppe gehörige Transformation sehon ein Doppelelement hätte, was nicht möglich ist, wenn $n>3$ ist*).

3) Es hann aber vorkommen, dass der Büschel $A\left(a_{1} a_{2} \cdots a_{n}\right)$ nicht ans $n$, sondern aus weniger von einander verschiedenen Strablen besteht. Nun kann auf einem Strahle $A a_{i}$ nicht anch der Punkt $a_{i+1}$ liegen. Denn sonst würde $T$ zeigen, dass auf dem Strahle $A a_{i+1}=A a_{i}$ auch $a_{i+2}, a_{i+3} \cdots$ lägen, d. h., dass er die ganze Gruppe enthaltel würde, einen Fall, den wir ausgeschlossen haben. Fis missen also $4 a_{4}$ und $A a_{2}$ verschieden sein. Augenommen es wären uar $A a_{1}, A a_{2}$ $\cdots A a_{q}$ unter sich verschieden, so würde gemüss den Erörterungen in No. 1) die Transformation $T$ den Buschel $A\left(a_{1} a_{z} \cdots a_{q}\right)$ in $A\left(a_{2} a_{3} \cdots a_{1}\right)$ überführen. Aber auch diese Gruppe ist eine cyllische und folglich existirt kein-sich selbst entsprechender Strahl, ausser wem $q=2$ ist, wobei die Transformation $T$ die Strahlen des Büschels $A$ involutorisch paart. In diesem Falle aber, der nar eintreten kann, wenn $n$ gerade, $=2 p$, ist, besteht die ganze Strahlengruppe $A\left(a_{1} \cdots a_{n}\right)$ nur aus zwei Strahlen $A a_{1}=g_{1}$ und $A a_{2}=g_{2}$, von welchen einer $g_{1}$ die Punkte $a_{1} a_{3} \cdots a_{z p-1}$, der zweite $g_{2}$ die Punkte $a_{2} a_{4} \cdots a_{z y}$, träht. $\mathrm{Da}$ die Transformation $T^{*}=1, i+2$ die Punkte $a_{1} a_{3} \cdots a_{z p-1}$ der Reihe nach in $a_{3} a_{5} \cdots a_{1}$ überfüht, so bilden diese Punkte auf $g_{1}$ eine cyklisch-projectivische Gruppe. Weil aber $T$ den Punkt $A$ ungeädert läst, gilt dasselbe bei $T^{2}$ und somit wird bei der, zu einer cykl.-proj. Gruppe einer Geraden gehörenden, Transformation $T^{*}$ ein Punkt $A$ dieser Geraden nicht verändert. Dies kann aber nur stattfixden, wenn die Anzahl der Punkte der Gruppe $<3$ ist, und verlangt folglich, dass hier $p=2$ set, wobei die ganze Gruppe aus $2 \cdot p-4$ Punkten bestehen würde. Da dieser Fall nicht eintreten kann, weil wir $n \geq 5$ angenonmen haben, so kann also niemals eine Doppellinie der Transformation dorch $A$ gehen.

4) Hieraus folgt einerseits, dass $y$ nicht durch $A$ gehen kann, andererseits, dass auf $g$ kein reeller Doppelpunt liegen kan; denn ein solcher würde eine durch $A$ gehende Doppellinie liefern. Dagegen liegen a uf $g$ zwei conjugirt inaginäre Doppelpunkte $B$ und $r$ der Transformation $T$.

Es sei nun $K_{1}$ der reelle Kegelschnitt, der in $B$ und $\Gamma$ die Linien $A B$ und $A T$ berihrt und durch den Puukt $a_{1}$ geht. Die Trapafornation $T$ führt diesen in einep Kegelschnitt $K_{2}$ uber, der ebenfalls $A B$ und $A T$ in $B$ und $\Gamma$ herüht, aber durch $a_{2}$ geht. Beide Kegelschnitte haben entweder, weil sie in denselben Punkten lieselben Gernden berühren, keinen weiteren Ponlat gemein, oder sie müssen ganz za-

- Vergl. Anulen BA. XI, Seite 90, Xo. 3. 
sammenfallen. Im letzteren Falle zeigt die Anwendung von $T$, dass auch $a_{3} \cdots a_{*}$ auf demselben Kegelschnitt $K_{1}$ liegen müssen. Liegt aber $a_{2}$ nicht auf $K_{1}$, sondern z. B. im Innern desselben, so muss, weil boide Kegelschnitte sich nicht schneiden, der ganze Kegelschnitt $K_{2}$ im Innern von $K_{1}$ liegen. Aus $a_{2}$ lüsst $T$ den Punkt $a_{3}$ hervorgehen, der dann im Innern von $K_{2}$ und daher auch von $K_{1}$ gelegen ist. Indem man so weiter schliesst, findet man, dass auch $a_{n}$ innerhalb $K_{1}$ gelegen ist und darans, dureh Anwendung der Umformung, dass $a_{1}$ im Innern von $K_{2}$ liegt. Dies wiederspricht der Annahme, dass $a_{2}$ und damit $K_{2}$ innerhalb $K_{1}$, also $a_{1}$ ausserhalb $K_{2}$ gelegen ist. Da man die Annahme, $a_{2}$ liege im Aeusseren von $\mathcal{K}_{1}$, genau ebenso wiederlegen kann, so ist bewiesen, dass alle Punkte der Gruppe auf einem Kegelschnitt liegen. Wir können also sagen: eine cylilisch-projectivische Gruppe von mindestens fünf Punlten in einer Ebene liegt entweder auf einer Geraden oder auf einem Kegelsehnitt.

5) Sei die Gruppe anf einem Kegelschnitt gelegen, $B$ irgend ein Punkt desselben, und $C$ der ibm in der projectivischen Beziehung entsprechende, so ist $B\left(a_{1} a_{2} \cdots a_{n}\right) \pi C\left(a_{2} a_{3} \cdots a_{1}\right) \nwarrow B\left(a_{2} a_{3} \cdots a_{1}\right)$ und es bildet also dann die Gruppe $a_{1} a_{2} \cdots a_{n}$ eine cyklisch-projectivische Gruppe von $n$ Punkten auf dem Kegelschnitt. In meiner früheren Arbeit (Bd. XI, Seite 95, No. 10) habe ich gezeigt, dass, wenn $n$ Punkte eines Kegelschnitts eine cykl.-proj. Gruppe bilden, sie auch noch in anderer Anordnung eine solche Gruppe bilden, in jeder nämlich, in welcher die Indices der Punkte eine arithmetische Reihe bilden. Sei $a_{1}^{\prime} a_{2}^{\prime} \cdots a_{n}^{\prime}$, eine derartige zweite Anordnung der Punkte, so giebt es für die Punkte des Kegelschnittes eine Transformation $\left|a_{i}^{\prime}, a_{i+1}^{\prime}\right|$. Man kann aber stets eine projectivische Umformung der Ebene eines Kegelschnittes in sich selbst herstellen, bei welcher der Kegelschnitt sich selbst entspricht, und die dessen Punkte nach demselben Gesetz verändert wie die gegebene Transformation. Bezeichnet man mit $a_{3}{ }^{\prime \prime}$ den Schnittpunkt der Tangenten in $a_{2}^{\prime}$ und $a_{2}{ }^{\prime}$, und nit $a_{3}$ " den der Tangenten in $a_{2}^{\prime \prime}$ und $a_{3}^{\prime \prime}$, so ist diese Transformation für unseren Fall

$$
\left|a_{1}^{\prime} a_{2}^{\prime} a_{3}^{\prime} a_{3}^{\prime \prime} ; \quad a_{2}^{\prime} a_{3}^{\prime} a_{4}^{\prime} a_{5}^{\prime \prime}\right|
$$

und diese stellt somit eine Transformation der Ebene dar, die durch $\left|a_{i}^{\prime}, a_{i+1}^{\prime}\right|$ bezeichnet werden kann. Folglich ist eine cykl.-proj. Gruppe in einer Ebene, nicht nor in einer, sondern in jeder Anordnug cyll.proj., in der die Indices der Punkte eine arithmetische Reihe bilden.

6) Die Transformation $T^{k}=|i, i+k|$ führt jeden Punkt der Gruppe in einen andern über, and lässt, wie $T$, den Punkt $A$ und die Gerade $g$ unverändert. Man kann aber zeigen, dass auch bei der Umformang $T^{k}, A$ der einzige nicht in $g$ gelegene Doppelpunkt ist. 
Ist $n$ ungerade, so sind nach dem in $\mathrm{Nr}_{4}$ 1) Benerkten alle Strahlen des Büschels $A\left(a_{1} a_{2} \cdots a_{n}\right)$ rop einander verschieden, weil auf jedem Strahle böchstens zwei Punkte liecten können, und bilden eine cykl.proj. Gruppe. Die Transformation $T$ * lisst aus ihnen die $A\left(a_{k+1} a_{k+q}\right.$ $\ldots a_{2}$ ) herrorgehen; dabei aber kann, wie Bd. XI, Seite $93, \mathrm{Nr} .8$, bewiesen ist, Kein Strahl unverändert bleiben. Ist dagegen $n$ gerade, $=2 q$, so ist $a_{1} a_{q+1}, a_{2} a_{q+2}, \cdots$ eine Involution auf dem Kegelschnitt, und es gehen $a_{1} a_{2+1}, a_{3} a_{q+2}, \cdots$ alle durch denselben Punkt. Weil aber durch die Umformung $T$ der Schnittpunkt von $a_{1} a_{1+1}$ mit $a_{2} a_{4}+2$ in den der Linien $a_{2} a_{s+q}$ und $a_{5} k_{q+3}$ übergeht, so entspricht der Mitcelpunkt der In rolution sich selbst, und ist also der Punlit $A$. Der Büschel $A$ enthält daher für $n=2 q$ nur die \$trahlen $A\left(a_{1} a_{7} \cdots a_{2}\right)$, die nach Nr. 1) eine cykl.-proj. Gruppe bilden. Die Cmformung $T$. liefert daraus die Strahlen $A\left(a_{3+1} a_{\phi-2} \cdots a_{q+2}\right)$; so lange diese von jenen verschieden sixd, kann kein Doppelstrabl existiren. Dagegen werden für $k=q$ beide Baschel identisch, and dann schneidet also der ursprüngliche und der abgeleitete Büschel die Linie $g$ in denselben Punkten, die sich selbst entsprechen müssen, Weil $n>5$ sein soll, ist $q \geq 3$, daher ist die $Z a h l$ der auf $g$ sich belust entsprechenden Punhte mindestens gleich drei, und folglich entspricht jeder Punkt von $g$, und damit jeder Strahl von $A$, sich selbst. Die Umformung $T^{q}$ ist sonach eine projectivische, und, weil die Puntite $a_{1}$ und $a_{7+1}$ sicb abwechselnd entsprechen, eine involutorische. Ausser dem Inrotionscentrum $A$ und den Punkten der Involutionsaxe $g$ konnuen aber keine Doppelemente existiren. In den vorher betrachteten beiden Fallen dagegen baun abterhaupt ausser $A$ kein weiterer Doppelpunkt existiren, weil sonst auch ein durch $A$ gehender Doppolstrahl vorhan. den sein würde.

T) Sei nun $\delta_{1}$ irgend ein anderer Punkt der Ebene, der aber nicht der Gruppe $a_{1} \cdots a_{n}$ angelört, noch auch mit $A$ zusanmenfallt oder auf $g$ liegt. Wenn die Umformungen $T, T^{2} \ldots$ aus ihm die Punkt: $b_{2} b_{3} \ldots b_{n}$ hervorgeben lassen, so hat man eine Gruppes $b_{1} b_{2} b_{5} \cdots b_{n}$ welche $T$ in $Z_{2} l_{3} \cdots b_{1}$ überfihrt. Keincr fisser Punlte kann mit cincm der Punte a identisech sein. Wäre nümlich $b_{k}$ etwa $=a_{k}$, sis wirde die Transformation $T$ nach $n-k+1$ maliger Anwendung aus $b_{k}$ den Punkt $b_{1}$ und aus $a_{i}$ einen anderen Paukt der Gruppe hervorgehen lassen, und diese miissten, unserer Annahme entgegen, identisch sein. Die. " Punkte $t$ milssen aber auch unter sich cerschieden sein, denn wenn $b_{k+1}=b_{1}$ wäre, so wäre auch $b_{k+2}=b_{2}$ a. s. w., und die Umformung $T^{k}$ w'ürde die Gruppe $b_{1} b_{2} \ldots b_{k}$ in $b_{1} b_{2} \ldots b_{k}$ äber. fuhren, so dass diese Pankte Doppelpunkte der Tranisformation $T^{k}$ wären. Dies ist nach dem Vorigen nur möglich, wenn Punkte von $g$ sind, und dies ist nicht der Fall, weil $b_{1}$ nicht auf $g$ liegen sollte. 
Es bilden also die Punkte $b_{1} b_{2} \ldots b_{\text {s }}$ eine neue cykl.proj. Gruppe von n Pankten, die durch dieselbe Transformation $T$ eyklisch vertauscht werden. Dies gilt anch noch, wenn $b_{1}$ der Linie $g$ angehört and erleidet in diesem Falle nur eine Ausnahme, wenn $n=2 q$ ist. Weil dann nämlich sehon die Unformung $T$ die Punkte von $g$ nicht ändert, besteht die aus $b_{1}$ hervorgehende Grappe nur aus $q$ Punkten.

8) Auf jede Gruppe, die anf die angegebene Art ans einem Punkte der Ebene abgeleitet wurde, lässt sich, wofern sie nicht gauz auf einer Geraden, und zwar dann auf $g$ liegt, die frühere Untersuchung anwenden, und so findet sich, dass jede solche Gruppe auf einem Kegelschaitt liegt, und dass alle diese Kegelschnitte in den imaginären Punkten $\mathrm{B}$ und $\Gamma$ von $g$ die Linien $A \mathrm{~B}$ und $A \Gamma$ berühren. Für alle diese Gruppen ist $g$ "die zu der Grappe gehörige Linie" im Sinne der Nr. 11) der fruhberen Abhandlung. Denn bezeichnet man mit $p_{1}$ den Schnittpunkt der Linien $a_{1} a_{n}$ und $a_{2} a_{n-1} \cdots$ mit $p_{2}$ den der Linien $a_{2} a_{n}, a_{3} a_{n-1} \cdots$, mit $p_{3}$ den der Linien $a_{1} a_{2}, a_{3} a_{n} \cdots$ und endlich mit $p_{i}$ den der Linien $a_{3} a_{1}, a_{4} a_{n} \cdots$, welche Punkte alle auf der zar Gruppe gehörigen Linie liegen, so entsprechen vermöge $T$ den Punkten $p_{1} p_{2}$ resp. die $p_{3} p_{4}$, und also die Linie $p_{3} p_{2}$ sich selbst. Da $g$ allein bei der Umformung $T$ sich selbst entspricht, so muss es die zur Gruppe gehorige Linie sein. Wählt man den Punkt $b_{1}$, von dem aus eine ueve Gruppe $b_{1} b_{2} \cdots b_{n}$ construirt werden soll, auf der Linie $A a_{1}$, so liegt $b_{2}$ auf $A a_{n}, \cdots b_{n}$ auf $A a_{n}$. Daraus folgt, dass man die sümmtlichen Gruppen erhält, deren Punkte durch die Transformation $a_{i} a_{i+1}$ cyklisch vertauscht werden, wenn man auf einem der erwähnten Kegelschnitte alle derartigen Gruppen construirt, und sie dann von $A$ aus auf alle bbrigen Kegelschnitte projicirt. Wird $g$ die unendlich ferne Gerade, so wird $A$ der Mittelpunkt von Kegelschnitten, die dieselben imaginären Asymptoten haben, also der Mittelpunkt von concentrischen und ähnlichen Ellipsen. Ein specieller fidl davon sind concentrische Kreise, auf welchen die Ecken der eingeschriebenen regulären $n$ Ecke die Punkte der Gruppen bilden.

\section{Punktgruppen im Raume.}

9) Indem wir uns zur Aufsuchung der cyklisch-projectivischen Punktgruppen $a_{1} a_{2} \cdots a_{n}(n \geq 6)$ im Raume wenden, suchen wir diejenigen Elemente des Raumes auf, welche bei der Transformation $T=|i, i+1|$ des Ranmes sich nicht andern. Es sind im Allgemeinen 13 versehiedene kalle möglich, die $\mathrm{v}$. Staudt in den Beiträgen zur Geometrie der Lage $\$ 35$. Seite 328 ff. beschrieben hat. Wir wollen hier ron diesen Resultaten keinen Gebranch machen, sondern nur den dort in Nr. 508 bewiesenen Satz benützen, dass zwei projectivische 
Räume jedenfalls einen reellen oder imaginären Punkt entsprechend gemein haben. Führt die Transformation $T$ eineu reellen Punkt in sich über, so führt sie anch den Strahlenbündel dieses Punktes in sich selbst uber. Wenn aber zwei projectivisch bezogene Strahlenbindel ineinander liegen, so haben sie sicher mindestens einen. Strahl entsprechend gemein. Folglich geht dann durch jenen Punkt ein reeller sich selbst entsprechender Strahl. Ist dayergen def Doppeipunkt ron $T$ imaginär, so muss sein reeller Träger sich selbst entsprechen. Denu da jedem reellen Punkt wieder ein solcher entsprechen soll, so muss dieser reellen Geraden wieder eine reelle Gerade entsprechen, und da sie ferner durch den Doppelpunkt gehen soll, so wird sie durch $T$ in die einzige, den Doppelpuakt enthaltende, reelle Gerade, näblich dessen reellen Träger, übergeführt, der sich also selbst zugeordnet ist. Wenn daher zioei Räume reell projectivisch siod, so haben sive jedonfalls eine reelle Gerade entsprechend gemein.

10) Sei nun $A$ diese jedenfills existirende Gerade, welche durch $T$ in sich übergeht (oder wenn es deren mehrere giebt, eine von ihnen). Wie oben in Nr. 3) kann man dann beweisen, dass durch $A$ nur dann eine Doppelebene gehen kapn (wenn nicht die ganze Gruppe in einer Ebene gelegen ist), wenn alle Punkte der Gruppe sich auf zwei Ebenen vertheilen, so dass aaf der einen $E_{1}$ die Punkte $a_{1} a_{3} \cdots$, auf der zweiten $E_{z}$ die Punkte $a_{2} a_{4} \ldots$ liegen, was unar bei geraden $n_{3}=2 p$, stattfiuden kann, und wobei dann $T$ die Ebenen $F_{1}$ und $E_{\text {? }}$ vertanscht. Dann ist aber die durch $T$ im Ebeuenbusehel $A$ herrorgebrachte Lmformung eine involutorische und existiren zwei Doppelebeuen, wenn überhaupt reelle vorhapden sind. Es sind also die betden Falle möglich:

a) Der Büschel $A$ hat zwei reelle Doppelebenen $E_{3}, E_{2}$, und alle Punkte der Grappe liegen anf zwei Ebenen, so dass von den $n=2 p$ Punkten auf jeder Ebene $p$ liegen; oder

b) Der Büschel $A$ hat heine reellen, sondern awei conjugirt imagiväre Doppelebenen.

11) Die Linie $A$ wird nun won jeder der Ebenen $a_{1} a_{2} a_{3}, a_{2} a_{3} a_{4} \ldots$ geschnitten. Denn würde eine von ihuen die Lipie gaviz enthalten, so würden sie alle $A$ enthalten, weil sie durch $T$ in einander übergehen, während $A$ angë̈ndert bleibt. Dann wäreu aber die beiden Ebenen $a_{1} a_{2} a_{3}$ und $a_{2} a_{3} a_{4}$ nicht verschieden (wenn wir annehtwen, dass keiner der Punkte der Gruppe auf $A$ liegt; und wenn einer auf $A$ lage, withde die ganze Gruppe auf $A$ liegen, und die ganze Gruppe würde eben sein. Bezeichnen wir aber den Schnittponkt der Ebene $a_{1} a_{2} a_{3}$ und der Linie $A$ mit $b_{2}$, den der Ebene $a_{2} a_{3} a_{4}$ und $A$ mit $b_{3} \ldots$... s. w., so geht die Punktreihe $b_{1} b_{2} b_{3} \ldots b_{3}$ durch die Umformung $T$ in die Reibe $l_{3} b_{3} \cdots b_{1}$ iber, und diese $n$ Pankte bilden 
also eine cyklisch-projectivische Gruppe. Die Anzahl der von einander verschiedenen Punkte dieser Gruppe ist sicher grösser als Eins. Denn wären alle Punkte mit demselben Punkte $b$ identisch, so wärden die Punkte $b a_{2} a_{3}$ gleichzeitig auf zwei Ebenen, also auf einer Geraden liegen müssęn, ebenso $b a_{3} a_{4}$, so dass $a_{2} a_{3} a_{4}$ auf einer Geraden lägen und die ganze Cruppe gerade wäre.

lst $q$ die Ancahl der verschiedenen Punkte, so kann man wie in Nr. 1) zeigen, dass $q$ ein Theiler von $n$ sein muss und dass diese $q$ Punkte wieder durch $T$ cyklisch vertauscht werden. Darans folgt, dass nur dann auf $A$ ein Doppelpunkt der Transformation liegen kann, wenn $q=2, n=2 p$ ist. Die Umformung vertauscht dann die beiden Punkte nnter sich, und ist also für die Linie $A$ eine involutorische, die entweder zwei oder keine Doppelpunkte besitzt. Hienach sind zwei Falle möglich:

c) Die Punktreihe $A$ hat zwei reelle Doppelpunkte, und alle Ebenen wie $a_{n} a_{1} a_{2}, a_{1} a_{2} a_{3}, \cdots$ der Gruppe gehen durch zwei Punkte von $A$, so dass von den $n=2 p$ Ebenen durch jeden Punkt $p$ gehen; oder

d) Die Punktreihe $A$ hat zwei imaginäre Doppelpunkte.

Aehnliches gilt natürlich für jede andere sich selbst entsprechende reelle Linie, die ausser $A$ noch vorhanden ist.

Wenn wir ron der Linie $A$ ausgehen, so kann jeder der beiden Falle von Nr. 10) mit jedem der beiden obigen sich combiniren, so dass im Ganzen 4 Möglichkeiten zu untersuchen sind, die wir mit den Zeichen ac, bc, ad und bd bezeichnen wollen.

12) Fall a c. Der Ebenenbüschel $A$ hat zwei reelle Doppelebenen $E_{1}$ and $E_{2}$, und die Punktreite zwei reelle Doppelpunkte $P_{1}$ und $P_{2}$. Jedem Punkt von $E_{1}$ z. B. wird durch $T$ wieder ein Punkt von $E_{1}$ zugeordnet. Wenn aber eine projectivische Umformung einer Ebene nur nwei Punkte $P_{1} P_{2}$ einer Linie $A$ ungeändert lāsst, so lässt sie entweder*) noch einen dritten Punkt der Ebene nnverändert; oder eine gerade Linie, die entweder durch $P_{1}$ oder durch $P_{2}$ geht und keinen weiteren Doppelpunkt enthält; oder eine gerade Linie, die entweder durch $P_{1}$ oder durch $P_{2}$ geht und in der jeder Punkt sich selbst entspricht. Wie aber in Nr. 10) bewiesen ist, kann eine sich selbst entsprechende Gerade, in dem hier vorliegenden Falle, entweder zwei oder keinen reellen Doppelpunkt enthalten, und folglich sind-die beiden zuletzt angefübrten Falle nicht statthaft. Somit muss die Ebene $E_{1}$ noch einen Doppelpunkt $P_{3}$ und ebenso die $E_{z}$ noch einen Doppelpunkt $P$, enthalten. Bei der Transformation $T$ bleibt also das Tetraeder

7) Vergl. v. Sta udt, Geom, d. Lage, Seite 169, Nr. 294 
$P_{1} P_{2} P_{3} P_{4}$ unverändert. Da durch jede hante zwe Doppelebenen der Transformation gehen, so muss pach der in Nr. 10) bei a) gemachten Bemerkung die ganze Gruppe auf zwei durch jene Kanten grebenden Ebenen liegen, von welchen eine die Pankte $a_{1} a_{3} \cdots$, die andere die $a_{2} a_{4} \cdots$ enthält. Da dies für jede Fante gilt, so müssen die Punkte $a_{1} a_{3} \ldots$ auf 6 Ebenen gelegen sein, yon welchen eine durch jede Kante geht, ond ebenso $a_{2} a_{4} \cdots$ anf 6 andern Ebenen. Dies geht nur an, wenn die ganze Gruppe aus den beiden Punkten $a_{1}$ und $a_{2}$ besteht.

13) Fall ad. Jede der beiden reellen durch $A$ gehenden Doppelebenen enthält $A$ als sich selbst entsprechende Gerade, auf der aber, weil der Fall d stattindet, kein reeller, sondern nur zwei imaginäre Doppelpunkte liegen. Folglich enthält jede der beiden Ebenen noch einen ausserhalb $A$ gelegenen reellen sich selbst entsprechenden Punkt. Die Verbindungslinie der beiden Punkte ergiebt eine zweite sich selbst entsprechende Gerade $B$.

Der Fall be ist mit diesem im Wesentlichen identisch. Der Büschel $A$ hat zwei conjugirt inaginäre Doppelebenen und die Punktreihe $A$ zwei reelle Doppelpunkte. Das Strahlbündel, welches einen dieser Punkte zum Centrum hat, entspricht sich selbst und enthält daher ausser der sich selbst entsprechenden Linie $A$ noch eine sich selbst zugeordnete Ebene. Diese kann nicht durch $A$ gehen, weil sie sonst, gegen die Aunahme, eine Doppelebene des Büschels $A$ wäre. Die beiden Doppelebenen, welche durch die beiden Doppelpunkte anf $A$ gehen, schneiden sich. folglich in einer sich selbst entsprechenden Linie $B$. Somit ist dieser Fall nur durch die Vertauschung von $A$ nad $B$ rom vorigen untersphieden upd reicht es aus, den ersten zu untersuchen. Weil durch $A$ swei Doppelebenen gehen, so liegen (Nr. 10) die Punkte $a_{1} a_{3} \ldots$ in einer Ebene $E_{1}$, und die $a_{7} a_{4} \ldots$ in einer zweiten Ebene $E_{3}$ durch $A$. Und weil die Linie $B$ awei sich entsprechende Punkte $P_{1}$ und $P_{2}$ trägt, die dureh die Ebenen $E_{1}$ und $E_{2}$ harmonisch getrennt werden, so gehen nach der bei $c$ in Nr. 11 ) bemerkten Eigenschaft die Ebenen $a_{n} a_{1} a_{2}, a_{2} a_{3} a_{4}, \ldots$ durch einen und die Ebenen $a_{1} a_{2} a_{3}, a_{3} a_{4} a_{5}, \cdots$ durch einen zweiten auf $B$ liegenden Punkt. Beide Eigenschaften verlangen, dass $n$ gerade $=2 p$ sei. Man lege nun von $P_{1}$ aus die Strahlen $P_{1} a_{3}, P_{1} a_{2}, \ldots P_{1} a_{n}$. Die Umformung $T$ führt diese der Reihe nach in $P_{1} a_{2}, P_{1} a_{3}, \cdots P_{1} a_{1}$ über, so dass sie eine cyklisch-projectivische Gruppe im Strahlenbüpdel $P_{1}$ bilden und folghich, wenn wir den Fall einer ebenen Gruppe ansschliessen, einem Kegel zweiter Ordnung $K$ angehören mússen, der alle Pankte der Gruppe enthält. Die Ebene $P_{1} A$ ist die zur Gruppe gebörige, weil sie sich bei der Transformation $T$ nicht ändert. Ein 2weiter Kegel mit gleichen Eigenschaften hat seine Spitze in $P_{2}$. 
14) Ds ergiebt sich hieraus folgende Construction einer solchen Gruppe. Anf einem Kegel mit der Spitze $P_{1}$ bestimme man eine cykl-proj. Gruppe von $n=2 p$ Strahlen $\alpha_{1} \alpha_{2} \cdots \alpha_{2 p}$. In der zu der Gruppe gehorenden Ebene $E$ nehme man eine beliebige Gerade $A$, die nicht durch $P_{1}$ geht, an und lege durch sie zwei Ebenen $E_{1}$ und $E_{2}$, die den Kegel in zwei Kegelschnitten treffen. Die eine dieser Ebenen, $E_{1}$, schneide man durch die Strablen $\alpha_{1} \alpha_{3} \cdots \alpha_{z p-1}$, die andere $E_{2}$ durch die Strahlen $a_{7} \alpha_{4} \cdots \alpha_{2 p}$, so sind die Schnittpunkte $a_{1} a_{3} \cdots a_{2 p-1}$ resp. $a_{2} a_{4} \cdots a_{2 y}$ die Punkte der Gruppe. Um den Beweis zu liefern, dass so eine cykl.-proj. Gruppe erhalten wird, nehme man auf der Polaren $B$ der Ebene $E$ in Bezug anf den Kegel den Punkt $P_{2}$ so an, dass er von $P_{1}$ durch die Ebenen $E_{1}$ und $E_{2}$ harmonisch getrennt ist und betrachte dann die projectivisehe Raumtransformation

$$
T^{\prime}=\left\{P_{1} P_{2} a_{1} a_{3} a_{5} ; P_{1} P_{2} a_{2} a_{4} a_{6}\right\}
$$

durch die $P_{1} P_{2}$ oder $B$ sich selbst entspricht. Da die Strahlen $P_{1} a_{1}$, $P_{1} a_{2}, \ldots$ einer cykl.-proj. Gruppe angehören, so schneiden sich die Ebenen $P_{1} a_{1} a_{4}$ und $P_{1} a_{2} a_{3}$ in einer Geraden $P_{1} p_{5}$, die der zur Gruppe gehörigen Ebene $E$ angehört*). In derselben Ebene liegt die Schnittlinie $P_{1} p_{6}$ der Ebenen $P_{1} a_{1} a_{3}$ und $P_{1} a_{2} a_{4}$, die Schnittlinie $P_{1} p_{7}$ der Ebenen $P_{1} a_{2} a_{3}$ und $P_{1} a_{3} a_{4}$, sowie die Schnittlinie $P_{1} p_{3}$ der Ebenen $P_{1} a_{2} a_{6}$ und $P_{1} a_{3} a_{5}$. Durch $T^{\prime}$ gehen aber die Linien $P_{1} p_{5}$ und $P_{1} p_{6}$ in resp. $P_{1} p_{2}$ und $P_{1} p_{\gamma}$ über, also die Ebene $E$ in sich selbst. Der Kegel mit der Spitze $P_{1}$, der durch die Strahlen $P_{1} a_{1}, P_{1} a_{3}, P_{1} a_{5}$ geht und in Bezug anf welchen $E$ die Polarebene von $B$ ist, wird also durch $T^{*}$ in einen Kegel umgeformt, der die Strablen $P_{1} a_{2}, P_{1} a_{4}$, $P_{1} a_{s}$ enthält und in Bezug auf den ebenfalls $E$ die Polarebene von $B$ ist. Durch beide Bedingungen wird aber derselbe Kegel bestimmt, derjenige nämlich, welcher der Construction za Grunde lag, und der sich also selbst entspricht. Da nun weiter die Ebene $a_{1} a_{3} a_{5}$ der Ebene $a_{2} a_{4} a_{6}$ entspricht und $E$ eine Doppelebene ist, so entspricht auch die Linie $A$ sich selbst. Nach der Construction ist der Wurf $A\left(P_{1} a_{1} P_{2} a_{2}\right)$ harmonisch, folglich $A\left(P_{1} a_{1} P_{2} a_{2}\right) \pi A\left(P_{1} a_{2} P_{2} a_{1}\right)$, und deswegen fuhrt $T$ die Ebene $E_{2}$ in $E_{1}$ über. Weil die Gruppe von Strablen $P_{1}\left(a_{1} a_{2} \cdots\right)$ eine cykl.-projectivische ist, so ist $P_{1}\left(a_{1} a_{3} a_{5} a_{2}\right)$ त $P_{1}\left(a_{2} a_{4} a_{6} a_{3}\right)$, und weil durch $T^{\prime}$ der Kegel sich nicht andert, so entspricht in dieser Umformung dem Strahle $P_{1} a_{2}$ der Strahl $P_{1} a_{3}$ und also dem Punkte $a_{3}$ der Punkt $a_{3}$. Auf diesem Wege weitergehend zeigt man, dass $T^{\prime}$ allgemein den Punkt $a_{i}$ in $a_{i+1}$ überführt, womit bewiesen ist, dass die construirte Gruppe die gewünschte Eigenschaft besitzt. 
15) Fall bd. Der Ebenenbüschel $A$ hat zwei conjungirt imaginäre Doppelebenen und die Punktreihe $A$ zwei conjungirt inaginärc Doppelpunkte. Seien $H_{1}$ and $H_{2}$ diese Ebenen und $\Pi_{1}$ und $\Pi_{2}$ die Doppelpunkte. Die Ebene $H_{1}$ wird durch $T$ in sich selbst abergefubrt und zwar so, dass die Punkte $\Pi_{1}$ und $\Pi_{2}$ ungeändert bleiben. Die Betrachtung der Fälle, die eintreten können, wenn eine Ebene projectivisch in sich trausformint wird ${ }^{*}$ ), zeigt daun, dass jedenfalls in $H_{1}$ noch eine imaginäre Doppellinie $\gamma$ existiren muss, welche dureh einen der beiden Punkte $\Pi_{1}$ oder $\Pi_{3}$, sagen wir durch $\Pi_{1}$, geht. Diese Linie $\gamma$ muss eine Gerade zweiter Art sein; denn wäre sie von der ersten Art, so hätte sie einen reellen Punkt, der der Ebene $H_{1}$ angehörte, ohne auf $A$ zu liegen, was nicht angeht, da $H_{1}$ inaginär sein soll. Im hier vorliegenden Falle können picht alle Punkte von $\gamma$ sich selbst entsprechen. Um dies $2 u$ beweisen, legen wir durch den Punkt $a_{1}$ der Grupne den einzigen reellen Strahl, der $\gamma$ schneidet. Entspricht dann der Schnittpunkt sich selbst, so entspricht anch jener Strahl, als sein reeller Träger, sich selbst und enthält daher den Punkt $a_{2}$. Derselbe Schluss zeigt aber, dass er auch $a_{3}$ enthalten muss u. s. w. Folglich kann die Annahme nur richtig sein, wenn alle Punkte der Gruppe anf einer Geraden liegen. Die Gerade $\gamma$ kana also entwede: ausser $\Pi_{1}$ noch einen zweiten Doppelpunkt enthalten, oder abur $\Pi_{1}$ ist der einzige auf ihr liegende Doppelpunkt.

Im zweiten Falle lege man durch $a_{1} a_{2} a_{3}$ die reellen Strahlen $g_{1} g_{2} g_{3}$, welche $\gamma$ scbneiden und mit dieser Geraden die imaginären Punkte $A_{1} A_{2} A_{3}$ gemein haben. Dureh die Transformation $T$ geht $a_{1}$ über in $a_{2}$, der $\gamma$ schneidende reelle Strabl $g_{1}$ also in den einzigen durch $a_{2}$ gehenden reellen Strahl $g_{2}$, welcher $\gamma$ schneidet, and daher auch, weil $\gamma$ sich selbst entspricht, $A_{1}$ in $A_{2}$; aus den gleichen Grunde geht $A_{3}$ hervor aus $A_{2}$. $D_{4}$ sun nach der Annahme die beiden projectivischen Punktreihen $\Pi_{1} A_{1} A_{4} \ldots$ und $\Pi_{1} A_{2} A_{3} \cdots$, von welchen die zweite aus der ersten vermöge $T$ hervorgeht, $\Pi_{1}$ allein entsprechend gemein haben, so ist der Wurf $\Pi_{4} A_{1} A_{4} A_{3}$ harmonisches) und deswegen sind die 4 reellen Träger $A g_{1} g_{2} g_{3}$ dur 4 Punkte Erzeugende einer Regelschaar und auf dieser $A g_{2}$ durch $g_{1} g_{3}$ harmonisch getronat Seien jetat weiter $y_{4} \cdots g_{4}$ die reellen Geraden, welche durch die Punkte $a_{4} \cdots a_{n}$ der Gruppe gehen und $\gamma$ schneiden, so folgt, weil $A g_{1} g_{2} g_{3}$ einer Regelschaar angehörẹ, vermbgte der Unformung $T_{\text {, }}$, dass auch $A g_{3} g_{3} g_{4}$ einer Regelschaar angehören, welche mit jener übereinstimmt, weil sie drei Gerade mit ihr gemein hat. Drurch wei-

*) v. Staudt, Beitr. 2. Geom. d. Lage, Seite 1\$6, Nr. 301.

* A. o. a. O. Seite 145, Nr. 241 .

**) A o. a. O. Seite 92, Yr. 143; diebe Anqalen Bd. VIII, Seite 175, Nr. 40. 
tere Anwendung desselben Schlusses ergiebt sich nun, dass alle Strahlen $A g_{1} g_{2} \cdots g_{n}$ Erzeugende einer Regelschaar sind, und ferner folgt, dass durch $T$ ans der Gruppe $A g_{1} g_{2} \cdots g_{n}$ die $A g_{2} g_{3} \cdots g_{1}$ hervorgeht. Folglich bilden die Linien $g_{1} g_{2} \cdots g_{n}$ eine cyklisch-projectivische Gruppe von Erzeugenden dieser Regelschaar und $A$ bleibt bei der zugehörigen Umformung ungeändert. Nan abberträgt sich aber der Satz, dass eine cylliscbe Transformation einer Geraden, die sich" auf mehr als zwei Punkte erstreckt, keinen Doppelpunkt besitzt, auch auf cyklische Transformationen der Erzeugenden einer Regelschaar (etwa indem man mit einem Leitstrahl schneidet) und zeigt so, dass eine Doppelerzengende $A$ nor existiren kann, wenn die Zahl der von einander verschiedenen der Linien $g_{1} g_{2} \cdots g_{n}$ zwei ist, von welchen beiden geraden Liniea dann jede die Hälfte der Punkte der Gruppe trägt. Ist dann aber $B$ diejenige Erzeugende der Regelschaar $A g_{1} g_{2}$, welche von $A$ durch $g_{1}$ und $g_{3}$ harmonisch getrennt ist, so ist $A g_{1} B g_{2} \pi A g_{z} B g_{1}$, d. h. $B$ entspricht durch $T$ sich selbst. $D a$ nun auf der Doppelgeraden $\gamma$ nur ein Punkt $B$ existirt, der von $\Pi_{1}$ durch $A_{1}$ und $A_{2}$ getrennt ist und dessen Träger in Folge dessen von $A$ durch $g_{1}$ und $g_{2}$ harmonisch getrennt ist, so muss $B$ der Träger ron $B$ und daher $B$ ein zweiter auf $\gamma$ gelegener Doppelpunkt sein. Da dies gegen unsere Annahme streitet, so ist diese nicht möglich.

16) Es bleibt also nur noch der Fall, dass $\gamma$ und damit $H_{1}$ noch einen zweiten Doppelpunkt $P_{1}$ enthält. Dann wird aber die conjungirt imaginäre Ebene $H_{2}$ den zu $P_{1}$ conjungirten Punkt $P_{2}$ als weiterèn Doppelpunkt tragen. Die Verbindungslinie $P_{1} P_{2}$ ist dann eine zweite reelle Doppelliaie. Dagegen sind die zwei Linien $\Pi_{1} P_{1}$ und $\Pi_{2} P_{2}$, sowie andererseits die beiden $\Pi_{1} P_{2}$ und $\Pi_{2} P_{1}$ conjungirt imaginär. Diese 4 Linien bestimmen mit dem Punkt $a_{1}$ der Gruppe ein einmanteliges Hyperboloid, ron dessen Erzengenden die der einen Schaar die Geraden $\Pi_{1} P_{1}$ und $\Pi_{3} P_{2}$, und die der anderen die Geraden $\Pi_{1} P_{2}$ und und $\Pi_{2} P_{1}$ sehueiden. Legt man nämlich durch $a_{1}$ und die beiden conjungirt imaginüren Linien $\Pi_{1} P_{1}$ and $\Pi_{2} P_{2}$ die reelle Gerade $g_{1}$, so geht durch jeden Punkt ron $g_{1}$ eine einzige Gerade, die die beiden conjungirten Geraden $\Pi_{1} P_{2}$ und $\Pi_{2} P_{1}$ schneidet, und zwar durch jeden reellen Punkt von $g_{1}$ eine reelle Gerade und alle diese gehören einer reellen Regelschaar*) $H_{1}$ an, welche jene zwei Paare conjungirt imagisärer Geraden gleichfalls enthält. Durch die Umformung $T$ geht diese Ftanche in ein auderes reelles Hyperboloid $H_{2}$ über, das durch den Punkt $a_{3}$ und dieselben vier imaginären Linien geht. Liegt $a_{2}$ auf $H_{1}$, so fallt $H_{2}$ mit $H_{1}$ zusammen, und die wiederholte Anwendung von $T$ 
lehrt, dass die ganze Gruppe der Fläche $H_{1}$ angehört. Kiegt aber $a_{2}$ nicht auf der Fläche, sondern z. B. im Aeussern von $H_{1}$, so folgt durch die Umformung, dass auch $a_{3}$ im Aeussern ron $B_{2}$ und folglich anch im Aeussern von $H_{1}$ liegt. Denn weil $H_{1}$ und $H_{2}$ die 4 imaginären Linien gemein haben, treffen sie sich in keipem reellen Punkt and somit liegt $H_{2}$ ganz susserhalb $H_{1}$, weil der Punkt $a_{2}$ von $H_{2}$ ausserhalb $H_{1}$ sich befindet. Es ergiebt sich dann weiter, dass nuch $a_{4}$ im Aensseren von $H_{2}$ und von $H_{1}$ sich befipdet u. s. w., bis man findet, dass $a_{n}$ die gleiche lage hat, woraus durch Anwendung von $T$ folgen würde, dass $a_{1}$ im Aensseren von $H_{2}$ gelegen ist; während wir annahmen, $a_{2}$ und damit $H_{2}$ sei im Aeusseren von $\tilde{H}_{1}$, und also $a_{1}$ im Inneren von $H_{2}$ gelegen. Ebensowenig kann $a_{2}$ im Inneren von $H_{1}$ sich vorfinden und muss daher, und mit ihm die ganze Grappe, der Fläche $H_{1}$ selbst angehören.

17) Legt man durch die Punkte der Gruppe die reellen Erzeugenden $g_{1} g_{2} \cdots g_{n}$ der einen Schaar und die $h_{1} h_{2} \cdots h_{n}$ der zweiten Schaar des Hyperboloids $H_{i}$, so führt $T$ diese, nach einem oben schon angewandten Schlusse, in $g_{2} g_{3} \cdots g_{1}$ resp. $h_{2} h_{3} \cdots h_{1}$ äber. Beide Gruppen bilden also cyklisch-projectivische Gruppen von Strahlen, die beide auch aus -weniger als $n$ Strahlen bestebeu können, wobei dann jeder Strahl mehr als eineu Punkt, aber jeder gleichviele Punkte tragen muss. Man kann also eine cyklisch-projectivische Grappe construiren, indem man die cyklischen Gruppen von Geraden $g_{1} g_{2} \cdots g_{n}$, $h_{1} h_{2} \ldots h_{n}$ aus den beiden Schaaren von Erzeugenden eines Hyperboloids herauswählt und $g_{1}$ mit $h_{1}, g_{2}$ mit $h_{2}, \cdots$ schneidet. Die Ranmtransformation nämlich, die die Punkte

in resp.

$$
g_{1} h_{1} \quad g_{1} h_{3} \quad g_{2} h_{1} \quad g_{2} h_{2} \quad g_{3} h_{3}
$$

$$
g_{2} h_{2} \quad g_{2} h_{3} \quad g_{3} h_{2} \quad g_{3} h_{3} \quad g_{4} h_{4}
$$

überführt, erzeugt aus dem durch die Geraden $g_{4} h_{1} g_{3} h_{2}$ und den Puakt $g_{3} h_{3}$ gehenden Hyperboloid das durch die Ceraden $g_{2} h_{2} g_{3} h_{3}$ und den Punkt $g_{4} h_{s}$ gehende, welches mit jenem identisch ist; der Linie $g_{3}$, weil sie durch den Punkt $g_{3} h_{3}$ und die beiden Geraden $h_{1} h_{2}$ gelegt ist, entspricht die Erzeugende, die durch $g_{4} h_{4}$ und die Geradell $h_{2} h_{3}$ geht, also $g_{4}$; ebenso der $h_{3}$ die Linie $h_{3}$. Entspricht der Linie $g_{4}$ die Linie $x$, so muss sein $g_{1} g_{2} g_{3} g_{4} \pi g_{2} g_{3} g_{4} x$. Es ist aber wegen der cykl.-proj. Gruppe $g_{1} g_{2} g_{3} g_{4} \backslash g_{2} g_{3} g_{4} g_{5}$, so dass $x$ mit $g_{3}$ ubereinstimmt; in gleicher Weise wird $h_{4}$ in $h_{3}$, also der Punkt $g_{4} h_{4}$ in den $y_{5} h_{3}$ ubergeführt. Man beweist ohne Weiteres in der Art, dass eine Transformation existirt, welche die Punkte der construirten Gruppe cyklisch vertauscht. Wenn die so gefundene Gruppe gane in derselben Ebene liegt, so ist $g_{1} g_{2} \cdots g_{n} \pi h_{1} h_{2} \cdots h_{n}$, und noggekehrt, wenn diese Beziehung besteht, liegt die Gruppe in einer Ebene. 
Alles zusammengefasst, ergiebt das Resultat: Eine eylilisch-projectivische Gruppe im Raume liegt entweder auf einer Geraden, oder auf einer Ebene, oder auf zwei Ebenen, oder auf einem Hyperboloide.

18) Nehmen wir an, eine eyklisch-projectivische Gruppe $a_{3} a_{2} \cdots a_{2}$ gehöre einer der letaten Arten an, und construiren dann, ausgehend von einem reellen Pankt $b_{1}$, der aber auf keinem der reellen Doppelelemente der Transformation $T$ liegt und mit keinem der Punkte $a_{1} a_{2} \ldots a_{n}$ identisch ist, die Punkte $b_{2}, b_{3}, \cdots b_{n}$, welche aus $b_{1}$ durch wiederholte Anwendung von $T$ hervorgehen. Die Anzahl der von einander verschiedenen ist sicher nicht $>n$. Wie in Nr. 7) kann man zeigen, dass keiner dieser Punkte mit einem der Punkte $a_{1} \cdots a_{n}$ zusammenfallen kann. Es kann aber auch die Anzahl der verschiedenen Punkte nicht $<n$ sein. Denn wäre sie nur $=k$, so dass $b_{k+1}=b_{1}$ wäre, so würde die Transformation $T^{k}$ die Punkte $a_{1} a_{2} \cdots a_{n}$ $b_{1} b_{2} \ldots b_{k}$ in $a_{k+1} a_{k+z} \cdots a_{k} b_{1} b_{2} \ldots b_{k}$ verwandeln und liesse demnach die Punkte $b_{1} b_{2} \ldots b_{k}$ unverändert. $\mathrm{Da}_{a}$ aber $T^{k}$ dieselben (reellen und imaginären) Doppelpunkte nicht ändert, die anch $T$ nicht ändert und deren Anzahl schon 4 beträgt, so würde ein weiterer Doppelpunkt, der nicht dem Tetraeder jener vier Pupkte angehörte, bewirken, dass die Umformung $T^{k}$ gar keinen Punkt änderte. Dies ist aber nur für $k=n$ möglich. Die neue aus $b_{1}$ entstehende Gruppe enthält daher ebenfalls $n$ Punlte. Aehnlich wie der in Nr. 7) bewiesene Satz kann auch dieser eine Ausnahme erleiden, wenn $b_{1}$ auf einer Doppelebene oder auf einer Doppelgeraden angenommen wird. Ich will auf die nähere Exörterung dieser Ausnahmen, sowie die damit zusammenhängende Frage, wann die Verbindungslinie zweier Punkte einer solchen Gruppe die beiden reellen Doppelgexaden schneidet, hier nicht weiter eingehen und nur bemerken, dass sie mit einer Eintheilung der cyklisch-projectivischen Gruppen in Unterarten zusammenhāngt, die mit der Eintheilung der regulären Polygone in Arten analog ist.

19) Auf die, wie oben gezeigt, aus $b_{1}$ entstehende neue Gruppe von $n$ Punkten lassen sich dieselben Sätze anwenden, wie auf die ursprungliche, und sie liegt also im Falle be oder a d auf zwei Ebenen und gleichzeitig auf zwei Kegeln; oder, im Falle bd, auf einem Hyperboloid. Im letaten Falle bilden alle, durch verschiedene Annahme fit $b_{1}$, so entstehenden einmanteligen Hyperboloide eine einfach unendliche Schaar von Flächen, die, weil sie 4 imaginäre Kanten eines Tetraeders enthalten, sich in keinem reellen Punkte treffen. Für alle Flïchen sind die Linien $A$ und $B$ conjugirte Polaren, weil die durch die eine gehenden imaginären Tangentenebenen, nämlich die Tetraederftachen, in Ponkten der andern berühren. Hat man anf einer der 
Flächen alle cyklisch-projectivischen Gruppen ron $n$ Punkten construirt, so kann man leicht die auf jeder andern finden. Liegen nämlich die beiden Punkte $a_{1}$ und $b_{1}$ zweier Gruppen auf einer Geraden, die $A$ and $B$ schueidet, so liegen, wie die Umformung $T$ lehrt, $a_{2}$ and $b_{2}$ ebenfalls auf einer solchen Geraden u. s. w. Man kanu also mit Hülfe solcher Strahlen die zweite Gruppe aus der ersten ableiten.

Ein specieller Fall wird gebildet durch die Schaar der concentrischen und coaxialen einmanteligen Rotationshyperboloide, wobei die Gerade $A$ die Rotationsase und die $B$ eine zur Axe senkrechte unendlich ferne Grade ist. Mit Hülfe von zwei regulären Vielecken, die man dem Kehlkreis einer dieser Flächen einschreibt, kann man dann die Erzeugenden beider Schăren zu cyklisch-projectivischen Gruppen ordnen und so leicht eine Punktgruppe realisiren.

Karlsruhe, 10. December 1877. 